\title{
Epigenetic Factors Altered in Dehisced Anther Correlated to Seed Dormancy in Paris polyphylla var. Yunnanensis
}

\author{
Xia Cheng1, Bin Wang1*\#, Linyun Liu1, Zhen Zhao², Xia Ling33, Feng Zhao¹, Dingkang Wang1\# \\ ${ }^{1}$ School of Agriculture and Life Sciences, Kunming University, Kunming, China \\ ${ }^{2}$ Kunming Dianchi Lake Environmental Protection Collaborative Research Center, Kunming University, Kunming, China \\ ${ }^{3}$ International Exchange and Cooperation Division, Kunming University, Kunming, China \\ Email: "wangbbiomed@126.com, "wdk117@163.com
}

How to cite this paper: Cheng, X., Wang, B., Liu, L.Y., Zhao, Z., Ling, X., Zhao, F. and Wang, D.K. (2019) Epigenetic Factors Altered in Dehisced Anther Correlated to Seed Dormancy in Paris polyphylla var. Yunnanensis. Agricultural Sciences, 10, 15171533.

https://doi.org/10.4236/as.2019.1012112

Received: October 28, 2019

Accepted: November 29, 2019

Published: December 2, 2019

Copyright $\odot 2019$ by author(s) and Scientific Research Publishing Inc. This work is licensed under the Creative Commons Attribution International License (CC BY 4.0).

http://creativecommons.org/licenses/by/4.0/

\begin{abstract}
Paris polyphylla var. yunnanensis (Franch.), one of the best-known medicinal plants in China, has a dehiscent anther which physiologically work in pollination, however, the dehiscent anther always closes in response to darkness every day, and watering or raining every time. To explore this frequently closing and its unkown physiology, next-generation sequencing was performed, and the transcriptome was de novo assembled. RNA-sequencing was carried out in 15 samples including seven openning samples, four closed samples owing to darkness or watering, and tissue samples (leaf, petal, calyx, and stigma) were used for control. We obtained 72.75 GB data, assembled into 79,815 unigenes. Differentially expressed unigenes (DEGs) between opened and closed anther samples were 6231 and the DEGs between anther and control samples were 2831. Comparation between the two DEGs by KEGG enrichment showed that "plant hormone signal transduction" pathway is the most significant pathway for DEGs from closing anther vs. opening anther, and expression model of DEGs in the pathway might elicit change in germination and seed dormancy. Further examination of the action of the signal pathway on physiology showed "chromatin binding" function was prominent in "DNA binding" function of annotated DEGs between opened and closed anthers, of the 215 "chromatin binding" unigenes, 120 were involved in epigenetic silencing, and 50 of the epigenetic unigenes were directly related to germination or seed dormancy, strongly correlating anther closing to epigenetic modification and seed dormancy. These results were verified that at least three auxins involved in seed dormancy showed same expression patterns occurred in abnormal closing anther and seed embryo in Paris
\end{abstract}

${ }^{*}$ Bin Wang contributed equally as the first author. 
polyphylla var. yunnanensis. In conclusion, the information from transcriptome point out that frequent abnormal closing of dehiscent anthers possibly transfer the impact on seed dormancy, and epigenetic modification happened in closing may be the cause.

\section{Keywords}

Abnormal Closing, Dehiscent Anther, Epigenetic Modification, Paris polyphylla var. Yunnanensis, Seed Dormancy

\section{Introduction}

Paris polyphylla is a temperate genus of flowering plants belonging to the family Trilliaceae that includes 24 species distributed throughout Europe and East Asia. Paris is native to Southwest China [1], and the Yunnan-Guizhou Plateau is considered the center of its diversity [2] [3]. As one of the most well-known medicinal plants in China, $P$. polyphylla var. yunnanensis is highly prized for its pain-relieving and anti-inflammatory properties [4] [5] [6]. Recent studies have shown that P. polyphylla also has anticancer activity [7] [8].

From over-collection during the past decades, wild resources have become scarce, and P. polyphylla is now considered an endangered species. In fact, its conservation is only possible through natural propagation because asexual reproduction via tissue culture remains a challenge [9]. The rate-limiting factors in the cultivation of $P$. polyphylla is prolonged dormancy (18 months) and slow growth from seed (3 - 4 years) [10] [11]. Thus, understanding the mechanism of prolonged seed dormancy in this species is key to accelerating its propagation.

Previously, we observed that the anthers of $P$. polyphylla closed in the evening and reopened in the morning, with the entire dehiscence period lasting up to 20 days [12]. Indeed, alterations in darkness, lower temperature, and humidity can drive the closing and reopening of dehiscent anthers [13]. Edwards et al. [14] reported that the dehisced anthers of Lilium philadelphicum closed when it rained, and they proposed that effective anther closing is an adaptation to protect pollen during the flowering period. However, as revealed by our previous study results [15], the anther dehiscence period of $P$. polyphylla var. yunnanensis can last up to 23 days, but pollen viability peaks on day 1 and gradually decreases, and the receptivity of the stigma to pollen peaks on days 11 to 13 . Within this short period, the occurrence of anther closure at night and during rain can shorten the pollination period and seriously affect normal fertilization. However, little is known about the molecular mechanisms underlying the sensitivity or ability of dehiscent anthers to close in response to the environment, and whether and how the genetic mechanism of the closure of anthers of $P$. polyphylla affects further physiological activity.

In this study, RNA-sequencing (RNA-Seq) technology was used, and the 
transcriptome of $P$. polyphylla anther was de novo assembled. Furthermore, the differential expression of unigenes (DEGs) between closed and opened anthers was analyzed. The transcriptomic data can improve our understanding of the mechanism underlying abnormal closure of anthers in flowering plants.

\section{Materials and Methods}

\subsection{Plant Material}

The seeds of $P$. polyphylla var. yunnanensis were harvested from plants grown in Yunnan Province, China in October 2012, and the anther and other tissue samples were collected in March 2017. Paris Polyphylla plants were grown at an altitude of $1995 \mathrm{~m}$, with an annual average temperature $14.26^{\circ} \mathrm{C}$ and an annual average rainfall of $957.12 \mathrm{~mm}$, in the germplasm resource garden of the Medicine Research Institute, which affiliate to Yunnan Academy of Agricultural Sciences, located in County of Songming, Kunming City, China. Fifteen samples, including seven closed and four opened samples, were collected from three plants. Furthermore, four tissue samples, including leaf, petal, calyx, and stigma were collected to analyze the changes in anther (Table 1). All tissue samples were immediately frozen in liquid nitrogen and stored at $-80^{\circ} \mathrm{C}$ until RNA extraction.

\subsection{RNA Extraction and Library Preparation}

For Illumina sequencing, the total RNA was extracted using the RNeasy plant kit (BioTeke, Beijing, China) and quantified using an Agilent 2100 Bioanalyzer (Agilent technologies, USA). The total RNA concentration of all the samples was equal to or greater than $400 \mathrm{ng} \cdot \mu \mathrm{L}^{-1}$; the $\mathrm{OD}_{260 / 280}$ ranged from 1.8 to 2.2; the RNA 28S:18S was higher than 1.0; and the RNA integrity number was higher than 7.0. The RNA samples were enriched with NEBNext Poly (A) mRNA

Table 1. List of different samples of $P$. polyphylla with anther status.

\begin{tabular}{ccc}
\hline Sample & plant & Status \\
T7 & plant 1 & Never opened \\
T1 & plant 2 & Before opening in morning \\
T2 & plant 2 & Semi opened \\
T3 & plant 2 & Opened \\
T4 & plant 2 & Closing initiation \\
T5 & plant 2 & Semi closed \\
T6 & plant 2 & Fully closed \\
T8 & plant 3 & Opened before watering \\
T9 & plant 3 & Closed after watering \\
T10 & plant 3 & Semi opened after watering \\
T11 & plant 3 & Fully opened after watering
\end{tabular}


Magnetic Isolation Module (New England Biolabs, USA), and then a cDNA library was constructed using NEBNext mRNA Library Prep Master Mix for Illumina (New England Biolabs, USA) and NEBNext Multiplex Oligos for Illumina (New England Biolabs, USA). The length of each cDNA fragment was verified by $1 \%$ agarose gel electrophoresis and quantified by the real-time PCR using the KAPA Library Quantification Kit by Illumina GA Universal (KAPA, USA). The quantified library was used for cluster generation with an Illumina cBot (Illumina Inc., USA), and then sequenced on an Illumina $\mathrm{HiSeq}^{\mathrm{TM}} 2500$ (Biomarker Biotechnological Co., Beijing, China).

\subsection{Illumina Reads Processing and de Novo Assembly}

The original paired-end reads were filtered to obtain the "clean reads" for de novo assembly. This process included the removal of reads with adaptor contamination or unknown nucleotides, and low-quality reads with ambiguous sequence "N." The "clean reads" from all samples of $P$. polyphylla were used for the de novo assembly using Trinity software [16]. This software assembles the reads into longer transcripts to form contigs, clusters the contigs according to paired-end information and similarity among contigs, and selects the main transcripts as unigenes.

\subsection{Gene Annotation and Analysis}

Functional annotations were performed by sequence comparison against public databases, including the NCBI NR (http://www.ncbi.nlm.nih.gov/), Swiss-Prot (http://www.expasy.ch/sprot/), COG (http://www.ncbi.nlm.nih.gov/cog/), and KEGG databases (http://www.genome.jp/keg/) using BLAST software ( $E$-value < $\left.1 \mathrm{e}^{-5}\right)$. The functional assignments were mapped to GO terms (http://www.geneontology.org/), and classification was performed using WEGO software (http://wego.genomics.org.cn/cgi-bin/wego/index.pl). The reads were mapped to the assembled unigenes using Bowtie [17], and their expression levels were estimated using the Expectation-Maximization (RSEM) software [18]. Gene expression level was determined by calculating the reads per kilobase per million (RPKM) for mapped reads [19].

\subsection{Analysis of Differentially Expressed Genes}

We detected the differentially expressed genes either using DESeq

(http://precedings.nature.com/documents/4282/version/2), when the samples fit the replication required for an analysis, or by using EBSeq [20]. BenjaminiHochberg method [21], using the FDR, was applied to correct the $P$ value of the original hypothesis. Here, The false discovery rate (FDR) was set to a threshold of $<0.01$ [22], and fold change was set to a threshold of $\geq 2$ as the criterion [23]. The unweighted pair-group method with arithmetic mean (UPGMA) cluster analysis was used to compare the relationship between differential unigene clusters. 


\section{Results}

\subsection{De Novo Assembly of the Anther Transcriptome}

After filtering out low-quality reads and vector sequences, we obtained 72.75 GB of data with 13,981,216 - 16,999,694 paired-end reads from each sample. Of the clean reads, more than $93 \%$ had Phred-scaled mapping scores equal to the corresponding error probability of $0.01(\mathrm{Q} 20)$ (Table 2). These results showed that the quality of these sequences was reliable. Trinity [16] was used to mix the samples and assemble a consolidated unigene library that included 12,083,102 contigs, 280,777 transcripts, and 79,815 unigenes. The N50 length of transcripts was $1215 \mathrm{bp}$ and the mean length of the unigenes was $1098 \mathrm{bp}$ (Table 3). Bowtie analysis [17] revealed that each read had a BLAST efficiency of $>62.19 \%$.

Table 2. Sequence quality report for 12 selected $P$. polyphylla samples.

\begin{tabular}{cccccccc}
\hline Sample ID & Read sum & Base sum & $\begin{array}{c}\text { GC } \\
(\%)\end{array}$ & $\begin{array}{c}\text { N } \\
(\%)\end{array}$ & $\begin{array}{c}\text { Q20 } \\
(\%)\end{array}$ & $\begin{array}{c}\text { Cycle } \\
\text { Q20 }(\%)\end{array}$ & $\begin{array}{c}\text { Q30 } \\
(\%)\end{array}$ \\
\hline T1 & $16,999,694$ & $3.43 \mathrm{E}+09$ & 50.27 & 0.01 & 98.75 & 100 & 93.42 \\
T10 & $14,332,384$ & $2.89 \mathrm{E}+09$ & 48.85 & 0.01 & 98.78 & 100 & 93.55 \\
T11 & $14,820,910$ & $2.99 \mathrm{E}+09$ & 49.20 & 0.01 & 98.75 & 100 & 93.42 \\
T2 & $16,265,835$ & $3.29 \mathrm{E}+09$ & 50.07 & 0.01 & 98.78 & 100 & 93.54 \\
T3 & $15,645,762$ & $3.16 \mathrm{E}+09$ & 49.36 & 0.01 & 98.77 & 100 & 93.52 \\
T4 & $15,701,026$ & $3.17 \mathrm{E}+09$ & 49.58 & 0.01 & 98.80 & 100 & 93.61 \\
T5 & $16,011,697$ & $3.23 \mathrm{E}+09$ & 49.64 & 0.01 & 98.79 & 100 & 93.58 \\
T6 & $15,683,182$ & $3.17 \mathrm{E}+09$ & 50.30 & 0.01 & 98.77 & 100 & 93.52 \\
T7 & $15,203,793$ & $3.07 \mathrm{E}+09$ & 50.23 & 0.01 & 98.76 & 100 & 93.52 \\
T8 & $15,400,569$ & $3.11 \mathrm{E}+09$ & 46.65 & 0.01 & 98.84 & 100 & 93.90 \\
T9 & $14,542,923$ & $2.94 \mathrm{E}+09$ & 49.20 & 0.01 & 98.78 & 100 & 93.56 \\
\hline
\end{tabular}

Table 3. Summary of the anther transcriptome of Paris polyphylla.

\begin{tabular}{ccc}
\hline Length (bp) & Total Number & Percentage \\
\hline $300-300$ & & $0 \%$ \\
$300-500$ & 39,270 & $49.20 \%$ \\
$500-1000$ & 22,417 & $28.09 \%$ \\
$1000-2000$ & 12,433 & $15.58 \%$ \\
$2000+$ & 5695 & $7.14 \%$ \\
Total number & 79,815 & \\
Total length (bp) & $64,710,737$ & \\
N50 length (bp) & 1098 & \\
Mean length (bp) & 810.76 & \\
\hline
\end{tabular}




\subsection{Closure Is an Abnormal Process of the Dehiscent Anther}

Dehisced anthers of $P$. polyphylla close every evening and reopen every morning; to study the mechanism of this closure phenomenon, the relevant samples were classified as follows: before opening in the morning (sample T1), semi opened (T2), opened (T3), closing initiation (T4), semi closed (T5), fully closed (T6), and never opened samples (T7). Furthermore, the samples treated by watering included the following: opened before watering (T8), closed after watering (T9), semi opened after watering (T10), and fully opened after watering (Table 1). To analyze the DEGs involved in the regulation of opening and closing of anthers by external stimulus, the opened and closed samples due to night, the T1, T2, and T3 samples, which represent opened samples; and the T4, T5, and T6 samples (Table 1), which represent closed samples, were compared. The results showed that 1528 DEGs between closing and opening $(\log 2 \mathrm{FC}>1.5)$ occurred, of which 1515 were up-regulated (mean RPKM value of 30.75) and identified as the night closing cluster. In comparison, up-regulated unigenes were expressed at low levels or not at all during dehiscence (mean RPKM value of 1.3), suggesting that DEGs in charge of anther closing are primarily the result of the up-regulation of these unigenes. (Figure 1(a)).

As the anthers of $P$. polyphylla can close when watering, a 10-minute watering treatment was carried out. After watering, the anthers moved from a fully opened stage to closed, and were divided into opening before watering (T8), water-closing (T9, T10), and opening after watering (T11). There were DEGs between opening before or after watering (T8 and T11) and closing after watering (T9, T10), then a total of 1220 DEGs were obtained and named as water-closing; of them 901 unigenes were up-regulated and 319 unigenes were down-regulated (FDR $<0.01$ ), and most of the 901 unigenes $(762,84.4 \%)$ overlap with closing cluster owing to night. The clusters of water-closing related unigenes and closing in evening contain 762 of the same unigenes (Figure 1(b)).

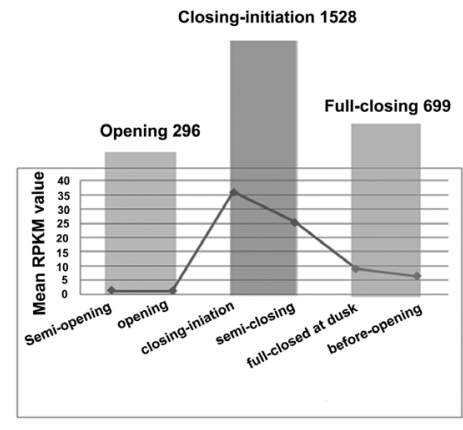

(a)

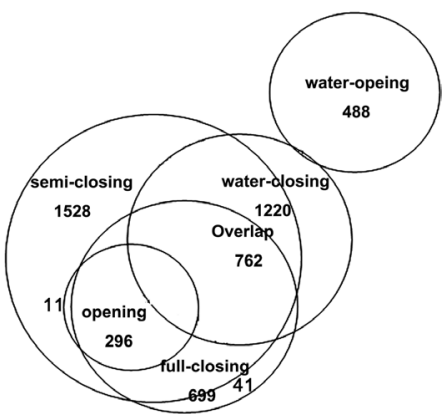

(b)

Figure 1. The differential expression unigenes between opening and closing. (a) Mean RPKM value of differential expression unigenes indicate the expression level closer to zero during the opening, close to 35 at the beginning of closing (closing-initiation), but retained the lower level when closing completely (full-closing); (b) The up-regulated unigenes in night-closing overlap with most of the DEGs occurring during watering-closing. 
Significantly enriched pathways (Fisher's exact test, $P<0.05$ ) revealed that during the transition from the open to closed stages (including night closing and watering-induced closing), the DEGs involved in the pathway were mostly up-regulated. Conversely, during the transition from closed to open stage, the genes involved in this pathway were mostly down-regulated. In addition, the common pathway for night closing and watering-induced closing is consistently involved in oxidative phosphorylation (energy generation) and ribosomes (protein translation), but the gene cluster for opening contained fewer unigenes involved in energy pathways, meanwhile, those unigenes involved in energy metabolism during opening were down-regulated (Table 4). These results suggest that closing of dehisced anthers is an energy dissipation process that requires the synthesis of related proteins. Conversely, the opening process does not require such high levels of energy and protein synthesis. That is, the closing of dehiscent anther is an abnormal stress response process and opening is a naturally restorative process, therefore, we inferred that closure is a response to withstand adverse stress.

\subsection{Abnormal Closing of Anther by External Stimulus Induces Epigenetic Silencing That Affects Seed Dormancy}

In order to analyze the DEGs involved in the regulation of opening and closing of anthers by external stimulus including night and watering, open samples T3 (opening in the morning), T8 (opening before watering) and T11 (opening after watering), and closed samples T1 (closing before opening in the morning), T6 (closing in the evening), T7 (never opened sample), and T9 (closing after watering) (Table 1), were compared. The clustering analyses based on the RPKM values showed that opening samples (T3, T8, T11) were prominently separated from the closest cluster (samples T1, T9, T6, T7). Differential expression analysis

Table 4. Expression variation of unigenes involved in the KEGG pathways at each status.

\begin{tabular}{|c|c|c|c|c|c|}
\hline differential KEGG pathway (p-value $<0.05$ ) & Semi closed & Fully closed & Opened & $\begin{array}{c}\text { Opened } \\
\text { after watering }\end{array}$ & $\begin{array}{c}\text { Closed } \\
\text { after watering }\end{array}$ \\
\hline Lysosome & Up & Up & Down & & \\
\hline Protein processing in endoplasmic reticulum & Up & Up & & & \\
\hline Oxidative phosphorylation & Up & Up & Down & & Up \\
\hline Proteasome & Up & & & & \\
\hline Ribosome & Up & Up & Down & & Up \\
\hline Phagosome & Up & Up & Down & & \\
\hline Drug metabolism-cytochrome P450 & Up & & & & \\
\hline Metabolism of xenobiotics by cytochrome P450 & Up & & & & \\
\hline Wnt signaling pathway & Up & & & & \\
\hline Diterpenoid biosynthesis & & & & Down & \\
\hline Sphingolipid metabolism & & & & Down & \\
\hline
\end{tabular}

Note: Up, upregulated; Down, down regulated. 
showed the DEGs between the two clusters is reliable. A total of 9819 DEGs were obtained from comparation of opened and closed anther samples; 4679 (47.7\%) unigenes were up-regulated and 5140 (52.3\%) unigenes were down-regulated. In total, 6231 (63.5\%) unigenes were annotated to KEGG, Swiss-Prot, and NR databases; among them, 3770 (60.5\%) unigenes were down-regulated with the mean RPKM value of 7.7, and 2462 (25.1\%) unigenes were up-regulated with the mean RPKM value of 30.75 .

Of the 6321 annotated DEGs, the unigenes involved in "response to external stimuli" were prominent in all biological processes (Figure 2(a)). Further analysis was carried out based on speculation that the external stimuli might transfer a signal to the nucleus, and finally induce "DNA binding" or "chromatin binding". In total, 215 unigenes were annotated to chromatin binding, the "chromatin binding" unigenes showed prominent in number (215 in 279, 77\%). Further analysis showed that among the 215 unigenes, 120 (56\%) unigenes (Figure 2(b)) are involved in silencing by epigenetic modification, including histone acetylation, histone H3-K9 methylation, histone lysine methylation, DNA methylation,

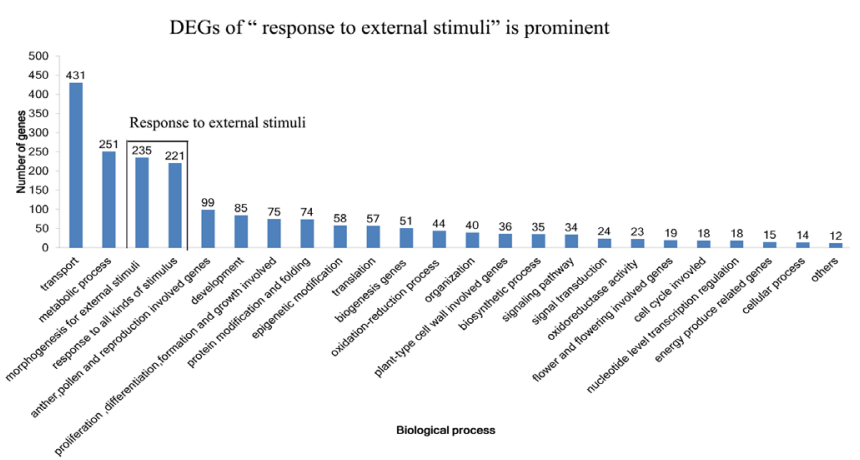

(a)

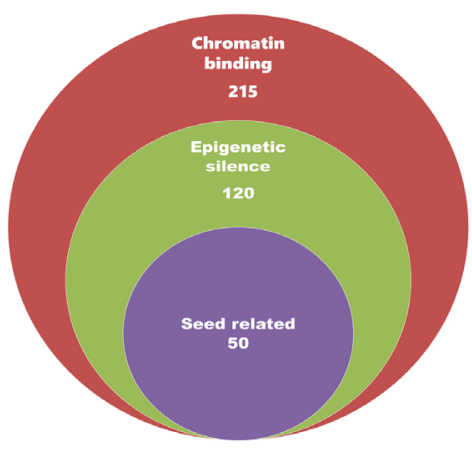

(b)

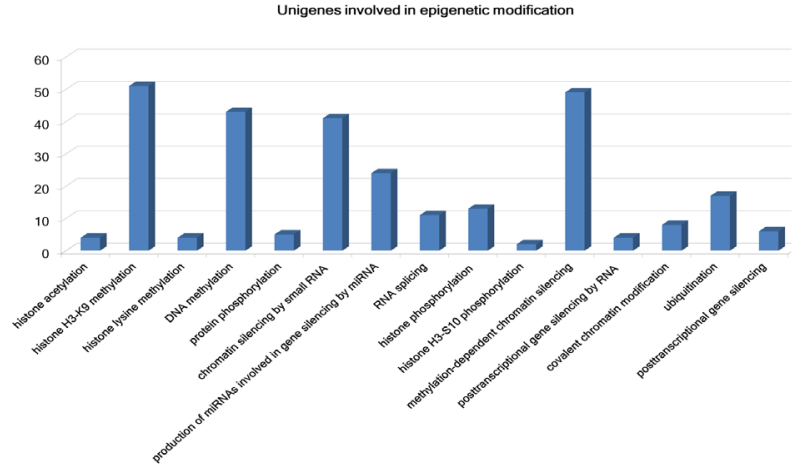

(c)

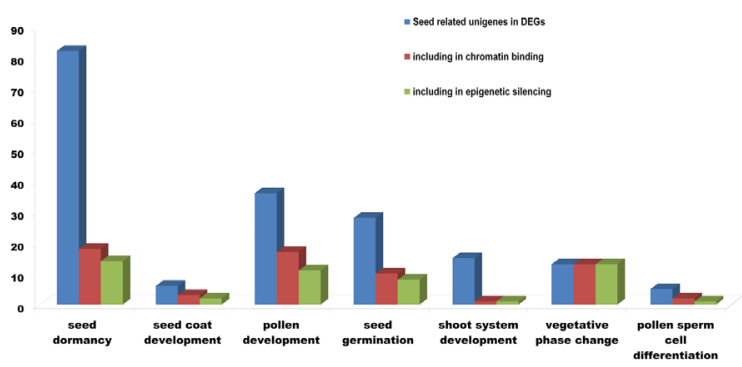

(d)

Figure 2. Functional annotation and classification of DEGs between opening samples (including biological repetition of T3, T8, and T11) and closed samples (including biological repetition of T1, T6, T9, and T10), involving responses to night and watering, the DEGs derived from duplicate values of comparation between three samples in "opening group" and each sample in "closed group". The results showed in (a) Function of "response to external stimuli" are prominent in the DEGs; (b) There were 215 unigenes annotated to "chromatin binding" function; (c) The 120 unigenes involved in "epigenetic modification" among the 215 unigenes with "chromatin binding" function, and (d) 50 epigenetic-involved unigenes showed seed dormancy or germinationrelated function. 
chromatin silencing by small RNA, miRNA synthesis involved in gene silencing by miRNA, RNA splicing, histone phosphorylation, histone H3-S10 phosphorylation, methylation-dependent chromatin silencing, posttranscriptional gene silencing by RNA, covalent chromatin modification, ubiquitination, and posttranscriptional gene silencing (Figure 2(c)). Interestingly, among the unigenes involving epigenetics, 50 were annotated to seed dormancy- or germination-related function simultaneously (Figure 2(d)). Moreover, 202 unigenes were located in mitochondria; among them, nine unigenes were involved in seed dormancy, germination, and development. These results showed that epigenetic modification genes have important roles in impacting seed dormancy, germination and development.

Furthermore, the results of KEGG pathways Enrichment analysis of DEGs between opening vs. closing and between anthers vs. control (leaf, petal, calyx, and stigma), showed the "plant hormone signal transduction" pathway is the most significant in former, but not in latter (Figure 3), and the variation in the expression of DEGs involved in the pathway analysis showed that the key genes, such as GID1, are up-regulated in the signal transduction to germination while closing, indicating down regulation of germination. Meanwhile, key genes, such as PYR/PYL and PP2C, which were down-regulated, and SnRK2 which up-regulated in the signal transduction were induced seed dormancy while closing (Figure 4), indicating a closing impact to seed dormancy through the "plant hormone signal transduction" pathway.

\subsection{Verification of Anther Closure Involved DEGs Are Correlated to Seed Germination}

To verify whether frequent abnormal closing of anthers affects biological processes that follow pollination and fertilization, such as seed dormancy and germination, the seed dormancy-related unigenes obtained from the mesodermal transcriptome of $P$. polyphylla var. yunnanensis [11] were compared with unigenes involved in anther closure (night closing and water-induced closing). The results revealed the significantly DEGs owning to anther closing including in the all type of seed dormancy-related unigenes found in embryo of seed, including phytohormone related, seed maturation related, cell wall growth related, circadian rhythms, flavonol biosynthesis related, cytochrome P450 and others. Among them, at leat four genes differential expression in anther closing showed similar expression pattern in embryo of seed (Table 5). interestingly, the four genes including three phytohormone related genes and one circadian rhythm gene. The three phytohormone related genes indicate similar expression pattern with showed in "plant hormone signal transduction" (Figure 4), where gibberellins up-regulated, abscisic acid down-regulated and brassinosteroid up-regulated, all which impact on seed dormancy or germination.

Furthermore, as a contrast, genes involved in the regulatory network related to the seed germination rate in Arabidopsis were used to retrieve in the "up-regulated" DEGs between closing and opening, finally, the retrieved unigenes 
Opening anther VS. closing anther

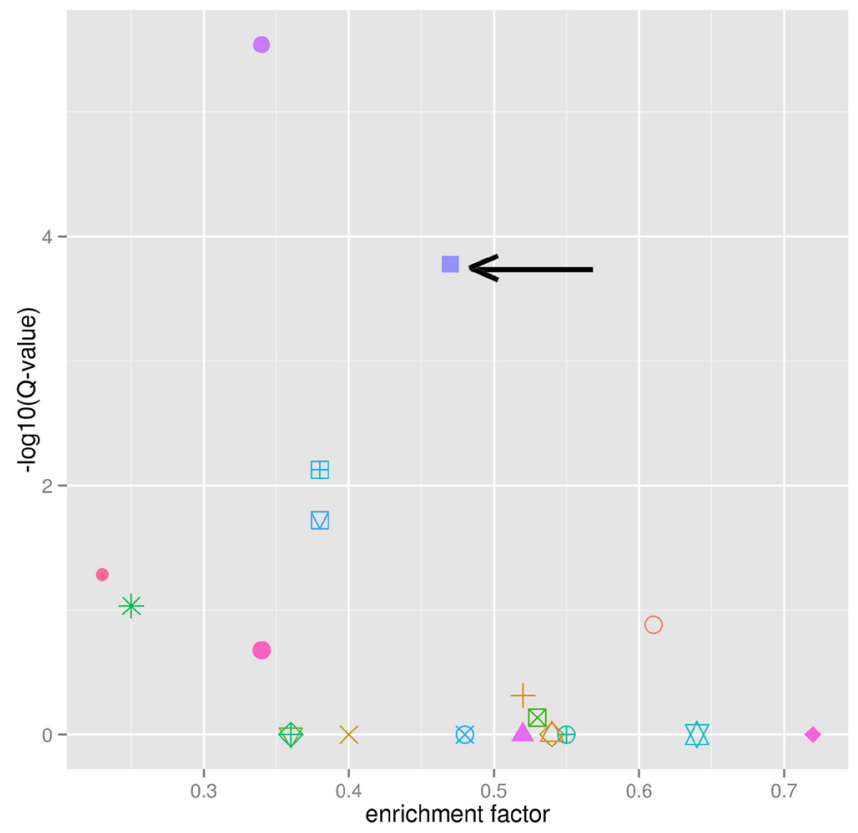

\author{
KEGG_pathway \\ Amino sugar and nucleotide sugar metabolism \\ $\triangle$ Biosynthesis of unsaturated fatty acids \\ + Carbon fixation in photosynthetic organisms \\ $X$ Circadian rhythm - plant \\ Fatty acid biosynthesis \\ $\nabla$ Flavonoid biosynthesis \\ $\bigotimes$ Glycerolipid metabolism \\ * Glycosphingolipid biosynthesis - globo series \\ $\diamond$ Glycosylphosphatidylinositol(GPI)-anchor biosynthesis \\ $\oplus \mathrm{N}$-Glycan biosynthesis \\ QX Phagosome \\ $\boxplus$ Phenylpropanoid biosynthesis \\ $叉$ Phosphatidylinositol signaling system \\ $\square$ Photosynthesis \\ Plant hormone signal transduction \\ Plant-pathogen interaction \\ $\triangle$ Sphingolipid metabolism \\ - Starch and sucrose metabolism \\ - Steroid biosynthesis \\ - Zeatin biosynthesis
}

Dehiscent anther VS. surrounding tissues ( leaf, petal, calyx, stigma)

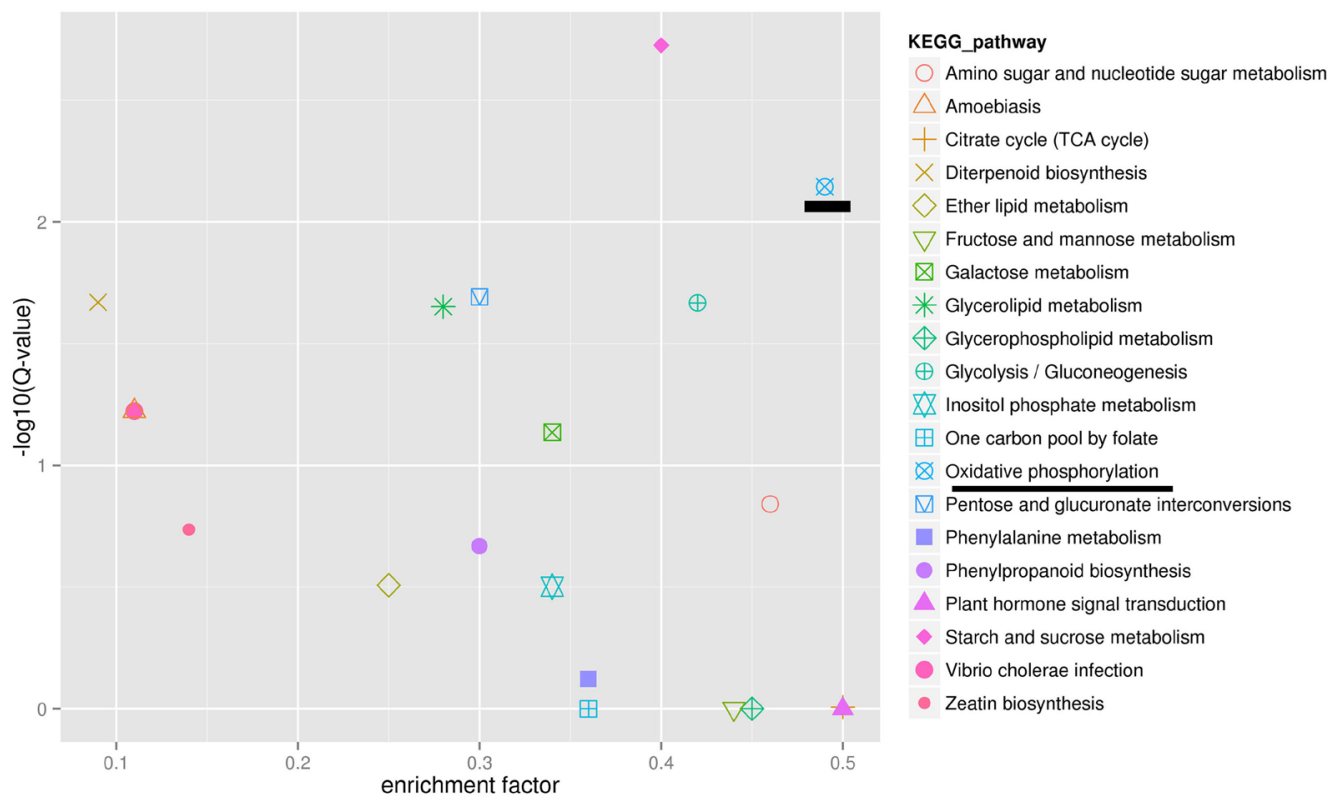

Figure 3. KEGG pathway enrichment scatter map between DEGs of opened samples vs. closed samples and anther samples vs. control (leaf, petal, calyx, and stigma). The arrow pointed to icons lie in the upper right corner showed "plant hormone signal transduction" pathway is the most significant one when closing anther compared with dehiscent anther; in contrast, the most significant one was Oxidative phosphorylation when dehiscent anthers compared with other tissues such as leaf, petal, calyx, and stigma. Note: The transverse axis is enrichment factor, which indicates the ratio of the DEGs to the proportion of the pathway entry in all the commented genes to the pathway entry. The enrichment factor is larger, indicating that the enrichment degree of the DEGs in the pathway is more obvious. The longitudinal axis is - $\log 10$ (Q-value), in which Q-value is the $\mathrm{p} \geq$ value after multiple hypothesis test, so the larger the $-\log 10$ (Q-value), the more significant the enrichment of DEGs in the pathway. So, the closer the entry is to the upper right corner, the greater the reference value is. 
Table 5. The seed dormancy-related unigenes shows similar expression pattern in embryo of seed which compared with endosperm, and in closing anther.

\begin{tabular}{|c|c|c|c|c|}
\hline Biological process & Gene bank description (gene name) & seed & Night-closing & water-closing \\
\hline \multirow[t]{8}{*}{ Phytohormone related } & Gibberellin 2-oxidase (GA2ox) & $3^{\mathrm{a}}$ & & $1\left(\right.$ down $\left.^{*}\right)$ \\
\hline & Abscisic stress ripening (ASR) & 5 & & 1 (down) \\
\hline & ABA INSENSITIVE 2 (ABI2); & 20 (up) & 2 (up) & 1 (down) \\
\hline & Auxin-repressed protein (ARP) & 8 (down) & & 2 (up) \\
\hline & $\begin{array}{l}\text { SAUR family protein (SAUR); Auxin response factor (ARF); } \\
\text { Auxin influx carrier component(AUX) }\end{array}$ & 18 & & 6 (up) \\
\hline & $\begin{array}{l}\text { IAA hydrolase (IAAH); IAA type protein; } \\
\text { Indole-3-acetic acid-amido synthetase (IAAS) }\end{array}$ & 13 (down) & & 2 (up) \\
\hline & $\begin{array}{c}\text { BRASSINOSTEROID INSENSITIVE } \\
1 \text {-associated receptor kinase } 1 \text { precursor (BRRK) }\end{array}$ & 3 (down) & & 1 (down) \\
\hline & Ethylene-responsive protein (ERP) & 7 & & 5 (down) \\
\hline \multirow[t]{5}{*}{ Seed maturation related } & $\begin{array}{l}\text { Seed maturation related Late } \\
\text { embryogenesis abundant protein (LEA) }\end{array}$ & 14 & & 2 (up) \\
\hline & Dehydrin (Dhn) & 13 & & 3 (down) \\
\hline & Lipid transfer protein precursor (LTP) & 6 & 2 (up) & 2 (up) \\
\hline & Lipid binding protein (LBP) & 5 & 3 (up) & 2 (up) \\
\hline & Ripening regulated protein DDTFR19 (TPS) & 10 & & 1 \\
\hline \multirow[t]{8}{*}{ Cell wall growth related } & Xyloglucan endotransglucosylase/hydrolase (XET) & 19 & 1 (up) & 3 (up) \\
\hline & Cell wall invertase (CIN) & 4 & 21 (up) & 5 (down) \\
\hline & Endo-beta mannanase (MAN) & 5 & & \\
\hline & Beta 1,3 glucanase & 14 & 2 (up) & 3 (up) \\
\hline & Pectin methylesterase (PME) & 18 & & 1 (up) \\
\hline & Expansins (EXP) & 8 & & 4 (up) \\
\hline & Polygalacturonase/inhibitor protein (PG/PGIP) & 36 & & 8 (down) \\
\hline & Sucrose synthase (SUS) & 22 (up) & & 2 (up) \\
\hline Cytochrome P450 & Cytochrome P450 (CYP) & 60 & 14 (up) & 21 (up or down) \\
\hline \multirow[t]{2}{*}{ Others } & Cell elongation protein (CEP) & 4 & 14 (up) & 9 (down) \\
\hline & Vaculoar H+-translocating inoraganic pyrophosphatase (VPP) & 10 & 2 (up) & \\
\hline
\end{tabular}

Notes: The expression pattern of seed dormancy-related unigenes in embryo of seed which compared with endosperm was referred to the paper of embryo transcriptome (Jianjun Qi, 2013); a means the number of unigenes; *up or down in the brackets mean the gene expression up-regulated or down-regulated. The overstriking words means the gene show similar expression pattern in embryo and closing anther.

could form a complete network, which induce from closing to seed germination (Figure 5). These network characterizing genes which might induce anther closing, including cell wall modifying genes, aquaporins, ion channels, and hormones. Cell wall structural proteins, including vegetative cell wall protein gp1, glycine-rich cell wall structural protein, and wall-associated receptor kinase 5 , were up-regulated in this gene cluster. The aquaporins including NIP1-1 and 


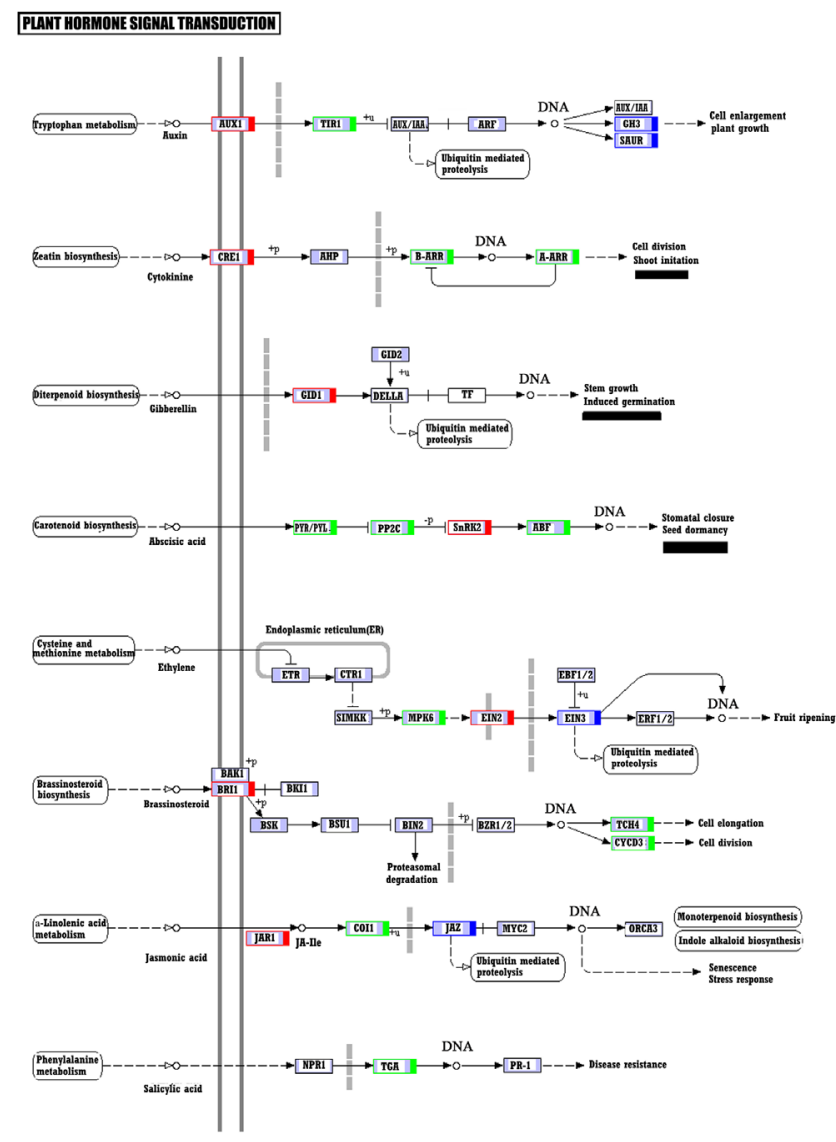

(a)
PLANT HORMOME SIGEAL IRANSDUGTION

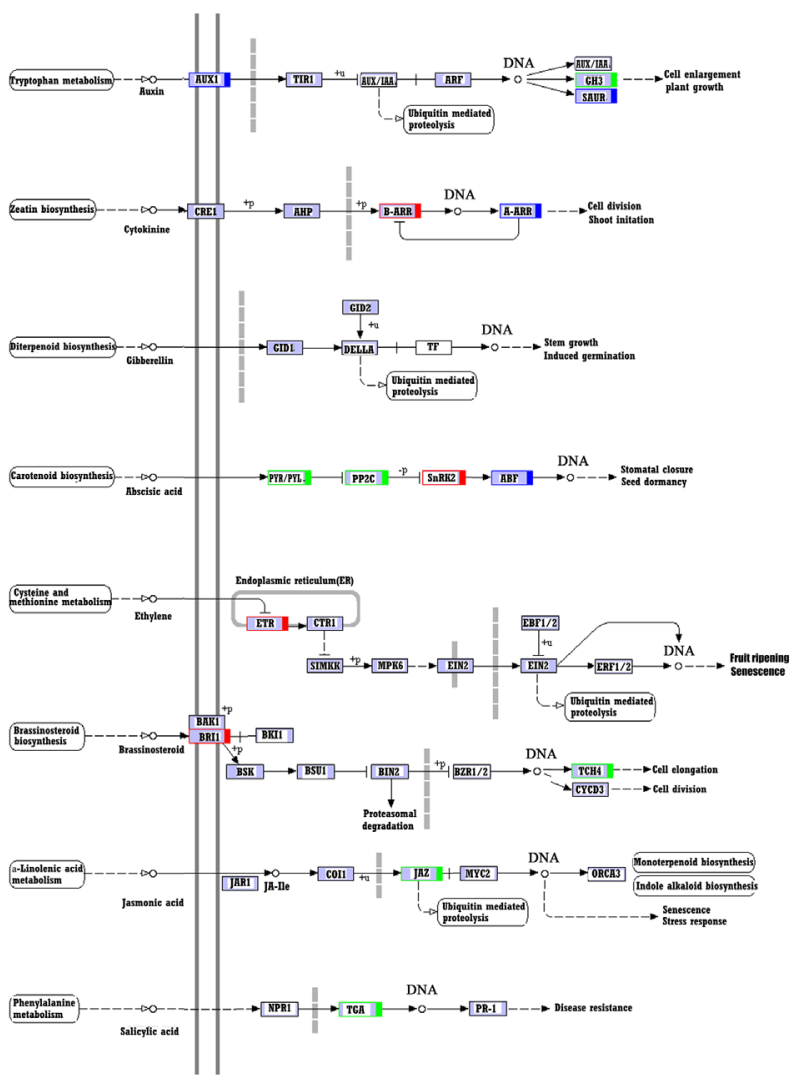

(b)

Figure 4. For the DEGs from opening to closing; the expression of genes involved in the "plant hormone signal transduction" pathway showed down-regulation in the signal transduction to germination, and up-regulation in the signal transduction to seed dormancy. However, for DEGs between anthers and control (leaf, petal, calyx, and stigma), the expression of genes involved in the "plant hormone signal transduction" pathway showed uncertain changes in germination or seed dormancy. Note: Red box indicates up-regulated genes; green box indicates down-regulated gene; blue box indicates simultaneous existence of up-regulated and down-regulated genes.

TIP2-1, voltage-dependent anion-selective channels, and chloride intracellular channel exc-4 were among the unigenes that were up-regulated during water-closing. Hormone-related genes up-regulated during anther closure were found to be related primarily to abscisic acid (ABA), jasmonic acid (JA), and gibberellin (GA), with concomitant strong up-regulation of transmembrane proteins, kinases, receptors, and molecules related to hormonal signal transduction. Indeed, these up-regulated genes are in regulatory pathways that can inhibit seed germination and prolong seed dormancy. For example, inhibition of germination via $\mathrm{ABA}$ and related signal transduction genes may involve strong up-regulation of superoxide dismutase and reduction of reactive oxygen species to inhibit germination. Up-regulation of cell wall-modifying enzymes and aquaporins can potentially inhibit seed germination. In addition, water-closing functions via increased expression of gibberellin 3-oxidase (GA3OX) and dihydroflavonol-4-reductase (DFR) can inhibit germination; moreover, water deprivation 


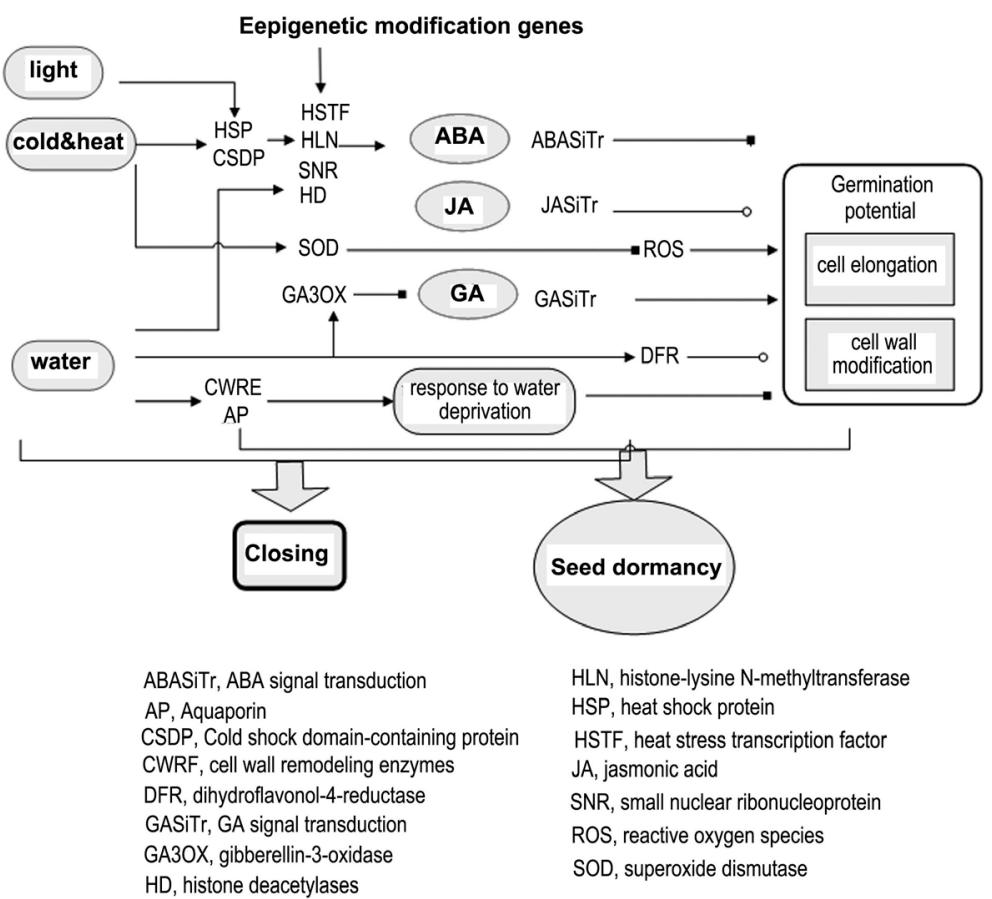

Figure 5. The up-regulated unigenes involved in anther-closing forming a regulatory network show impact from "light, temperature, and water" to seed germination and dormancy.

can inhibit germination via aquaporins. Furthermore, these networks also comprised many chromatin remodeling proteins including histone-lysine $\mathrm{N}$-methyltransferase (HLN), a small nuclear ribonucleoprotein (SNR), histone deacetylase (HD), and others. It is verified that up-regulation of these genes, especially chromatin remodeling genes, enable epigenetic modifications that can affect cellular processes following anther germination.

\section{Discussion}

Transcriptome sequencing has been used in several plant species and is considered an effective method to identify novel genes [19]. In the present study, we sequenced different anther stages of $P$. polyphylla using an Illumina paired-end sequencing platform and obtained DEGs between closed and opened anthers. After sequence annotation, we found that: 1) closure of dehiscent anthers is an abnormal process in response to external factors, such as night (darkness or decreased temperature) and water uptake, 2) this process consumes a large amount of energy, and 3) it is accompanied by abundant protein synthesis. Then closure is an abnormal process of the dehiscent anther.

As the opposite direction of dehiscence, the closing anther of P. polyphylla involves a high level of protein biosynthesis, including several types of cell wallmodifying enzymes and structural proteins, whose expression are either up-regulated or down-regulated. This indicates that the cellular mechanism of abnormal anther closure in P. polyphylla involves changes in the secondary wall. 
Meanwhile, the KEGG enrichment analysis revealed that the pathway "planthormone signal transduction" is the most significant, and the DEGs of the pathways revealed that gibberellins, brassinosteroid were up-regulated, while abscisic acid signal transduction was down-regulated. The cellular mechanism of anther dehiscence mainly involves changes in the endothecium, membranous tissue, and stomium that lead to thickening of the secondary inner wall of the anther [24]. Another wall thickening occurs due to the dehydration of pollen grains and stomium cells [25]. After the entry of $\mathrm{K}^{+}$into the pollen grain, the regulation of osmotic potential induces turgidity and extrusion of anther stomium tissue, thus, accelerating anther dehiscence [24] [26] [27]. In addition, plant hormones including jasmonic acid (JA), ethylene, and other growth factors, are important compounds that regulate anther dehiscence, and are important factors inducing responses in anther tissue cells to water deprivation [28]. Then, anther closing shows similar mechanism with dehiscence, in cell wall modification, hormones and ion flux, but they are different in detail, especially in type of hormones and ion flux. Indeed, gibberellins, brassinosteroid were up-regulated, while abscisic acid was down-regulated as DEGs occur in closing anther.

All the changes when closing owing to cell wall modification and cell elongation hormones [29] might impact germination potential, ultimately improving seed dormancy. But what change in anther which transfers to seeds is unclear. The epigenetic-related changes results from the involvement of mitochondriarelated genes which exclude energy production, and all types of epigenetic silencing including phosphorylation, methylation, acetylation, ubiquitination, miRNA synthesis involved in gene silencing, and mRNA splicing, should look as a hint to think over transferring of epigenetic marks. Importantly, some of these epigenetic-related unigenes are involved in regulation of seed dormancy, germination, and development. In the reckoning model refer to Arabidopsis, an regulatory network could formatted change from external stimuli to anther closing, and finally impact on seed dormancy, in which unigenes implicated in anther closure of $P$. polyphylla similarly include chromatin remodeling proteins, such as HLN, SNR, and HD. The HLN protein can form methylated histones, such as H3K9me2, which serves as a marker of gene expression regulation; SNR mediates the splicing of pre-mRNA by binding to the loop I region of U1-snRNA; $\mathrm{HD}$ is responsible for the deacetylation of lysine residues in the $\mathrm{N}$ terminal of the core histones (H2A, H2B, H3, and H4).

Further comparation analysis showed some known seed dormancy related genes in anther cloure occur similar expression pattern in embryo of seed. Importantly, these genes associate with regulation of three key hormone signal transduction pathways to seed dormancy. However, many questions still remain unclear, for example, if the epigenetic-related genes actually change the seed dormancy related genes? How the seed dormancy related genes expression changed in closing stage and finally keep the same expression pattern in seed? How many genes expression change in closure finally transfer to seed? 


\section{Conclusion}

Seed embryo of Paris polyphylla will stop at the globular stage for about 120 days after fertilization and seed germination requires 18 months for embryo development and release from dormancy, this long period of dormancy become the biggest obstacle for seedling. Although finer details of this mechanism for prolong dormancy should be elucidated by further studies, in the present study, the information from transcriptome of anther closing indicate that abnormal closing stimulated by night and water can lead to changes in a batch of epigenetic genes, and the epigenetic modification become the utmost cause for impacting seed germination, especially by change expression of seed dormancy related unigenes in abnormal closure and keep the same expression pattern in seed embryo.

\section{Funding}

This study was supported by the National Natural Science Foundation of China (31760256 to SH; 31260037 and 31760403 to SH) and Project of Talent introduction in Kunming university (code: YJL17003).

\section{Conflicts of Interest}

The authors declare no conflicts of interest regarding the publication of this paper.

\section{References}

[1] Li, H. (1984) The Phylogeny of the Genus Paris L. Acta Botanica Yunnanica, 6, 351-362. (In Chinese)

[2] Li, H. (1997) Trilliaceae. In: Institute Botanicum Kunmingense Academiae Sinicae, Ed., Flora Yunnanica, Science Press, Beijing, Vol. 8, 651-670. (In Chinese)

[3] Li, H. (1998) The Genus Paris L. (Trilliaceae). Science Press, Beijing, 83-84. (In Chinese)

[4] Faegri, K. and Van, D.P.L. (1979) The Principles of Pollination Ecology. Pergamon Press, Oxford. https://doi.org/10.1016/B978-0-08-023160-0.50020-7

[5] Hoekstra, F.A. (1983) Physiological Evolution in Angiosperm Pollen: Possible Role of Pollen Vigour. In: Mulcahy, D.L. and Ottaviano Pollen, E., Eds., Biology and Implications for Plant Breeding, Elsevier, New York, 35-41.

[6] Primack, R.B. and Kang, H. (1989) Measuring Fitness and Natural Selection in Wild Plant Populations. Annual Review of Ecology and Systematics, 20, 367-396. https://doi.org/10.1146/annurev.es.20.110189.002055

[7] Li, Z.H., Wan, J.Y., Wang, G.Q., Zhao, F.G. and Wen, J.H. (2013) Identification of Compounds from Paris polyphylla (Chonglou) Active against Dactylogyrus Intermedius. Parasitology, 140, 952-958. https://doi.org/10.1017/S0031182013000139

[8] Jia, X.B., Feng, L. and Wang, R.P. (2013) The Anti-Lung Cancer Activities of Steroidal Saponins of $P$. polyphylla Smith var. Chinensis (Franch.) Hara through Enhanced Immunostimulation in Experimental Lewis Tumor-Bearing C57BL/6 Mice and Induction of Apoptosis in the A549 Cell Line. Molecules, 18, 12916-12936. https://doi.org/10.3390/molecules181012916 
[9] Wang, Y.H., Jiang, T.T., Wei, F.U., et al. (2012) Tissue Culture of Paris thibetica. Lishizhen Medicine and Materia Medica Research, 23, 2014-2016.

[10] Chen, C., Yang, L.Y., Lv, L.F., Zhao, Q. and Yuan, L.C. (2007) Study on Seedling Techniques of Paris polyphylla var. yunnanensis. China Journal of Chinese Materia Medica, 32, 1979-1983.

[11] Qi, J., Na, Z. and Bing, Z. (2013) Mining Genes Involved in the Stratification of Paris polyphylla Seeds Using High-Throughput Embryo Transcriptome Sequencing. BMC Genomics, 14, 358-358. https://doi.org/10.1186/1471-2164-14-358

[12] Wang, D.K., Sun, G.F., Wang, L.F., Zhai, S.H. and Cen, X.J. (2009) A Novel Mechanism Controls Anther Opening and Closing in Paris polyphylla var. Yunnanensis. Chinese Science Bulletin, 54, 244-248.

https://doi.org/10.1007/s11434-008-0507-y

[13] Wang, D.K., Sun, G.F., Chen, X., Guo, L.H., Wang, L.F. and Guo, Z.M. (2008) Observation on the Anther Opening and Closing Phenomena of Several Species of Paris Genus. Journal of Anhui Agricultural Sciences, 36, 3709-3710, 3722.

[14] Edwards, J. and Jordan, J.R. (1992) Reversible Anther Opening in Liliumphiladelphicum (Liliaceae): A Possible Means of Enhancing Male Fitness. American Journal of Botany, 79, 144-148. https://doi.org/10.1002/j.1537-2197.1992.tb13631.x

[15] Wang, D.K., Sun, G.F., Zhai, S.H., Wang, L.F. and Guo, Z.M. (2008) Pollen Vitality and Stigma Receptivity of Paris polyphylla var. yunnanensis and P. mairei (Trilliaceae). Guihaia, 28, 443-446.

[16] Grabherr, M.G., Haas, B.J., Yassour, M., Levin, J.Z., Thompson, D.A., Amit, I., Adiconis, X., Fan, L., Raychowdhury, R., Zeng, Q., et al. (2011) Full-Length Transcriptome Assembly from RNA-Seq Data without a Reference Genome. Nature Biotechnology, 29, 644-652. https://doi.org/10.1038/nbt.1883

[17] Langmead, B., Trapnell, C., Pop, M. and Salzberg, S.L. (2009) Ultrafast and Memory-Efficient Alignment of Short DNA Sequences to the Human Genome. Genome Biology, 10, R25. https://doi.org/10.1186/gb-2009-10-3-r25

[18] Li, B. and Dewey, C.N. (2011) RSEM: Accurate Transcript Quantification from RNA-Seq Data with or without a Reference Genome. BMC Bioinformatics, 12, 323. https://doi.org/10.1186/1471-2105-12-323

[19] Garg, R., Patel, R.K., Tyagi, A.K. and Jain, M. (2011) De Novo Assembly of Chickpea Transcriptome Using Short Reads for Gene Discovery and Marker Identification. DNA Research, 18, 53-63. https://doi.org/10.1093/dnares/dsq028

[20] Leng, N., Dawson, J.A. and Thomson, J.A. (2013) EBSeq: An Empirical Bayes Hierarchical Model for Inference in RNA-seq Experiments. Bioinformatics, 29, 1035 1043. https://doi.org/10.1093/bioinformatics/btt087

[21] Benjamini, Y., Hochberg, Y. and Laron, Z. (1995) Mathematical Formulae for the Prediction of the Residual Beta Cell Function during the First Two Years of Disease in Children and Adolescents with Insulin-Dependent Diabetes Mellitus. Medical Hypotheses, 45, 486-490. https://doi.org/10.1016/0306-9877(95)90228-7

[22] Benjamini, Y. and Hochberg, Y. (2000) On the Adaptive Control of the False Discovery Rate in Multiple Testing with Independent Statistics. Journal of Educational and Behavioral Statistics, 25, 60-83. https://doi.org/10.3102/10769986025001060

[23] Mutch, D.M., Berger, A., Mansourian, R., Rytz, A. and Roberts, M.A. (2002) The Limit Fold Change Model: A Practical Approach for Selecting Differentially Expressed Genes from Microarray Data. BMC Bioinformatics, 3, 17. https://doi.org/10.1186/1471-2105-3-17 
[24] Hua, S.J., Meng, H.B., Wang, X.D. and Jiang, L.X. (2007) Cytological and Molecular Mechanism of Plant Anther Dehiscence. Chinese Journal of Cell Biology, 29, 389393.

[25] Keijzer, C.J. and Cresti, M. (1987) A Comparison of Anther Tissue Development in Male Sterile Aloe vera and Male Fertile Aloe ciliaris. Annals of Botany, 59, 533. https://doi.org/10.1093/oxfordjournals.aob.a087347

[26] Nelson, M.R., Band, L.R., Dyson, R.J., Lessinnes, T., Wells, D.M., Yang, C., Everitt, N.M., Jensen, O.E. and Wilson, Z.A. (2012) A Biomechanical Model of Anther Opening Reveals the Roles of Dehydration and Secondary Thickening. New Phytologist, 196, 1030-1037. https://doi.org/10.1111/j.1469-8137.2012.04329.x

[27] Pacini, E., Guarnieri, M. and Nepi, M. (2006) Pollen Carbohydrates and Water Content during Development, Presentation, and Dispersal: A Short Review. Protoplasma, 228, 73-77. https://doi.org/10.1007/s00709-006-0169-z

[28] Ishiguro, S., Kawai-Oda, A., Ueda, J., Nishida, I. and Okada, K. (2001) The Defective in Anther Dehiscience Gene Encodes a Novel Phospholipase A1 Catalyzing the Initial Step of Jasmonic Acid Biosynthesis, Which Synchronizes Pollen Maturation, Anther Dehiscence, and Flower Opening in Arabidopsis. Plant Cell, 13, 2191-2209. https://doi.org/10.1105/tpc.010192

[29] Farrooq, M., Sma, B., Khalid, M., et al. (2006) Nutrient Homeostasis, Metabolism of Reserves, and Seedling Vigor as Affected by Seed Priming in Coarse Rice. Canadian Journal of Botany, 84, 1196-1202. https://doi.org/10.1139/b06-088

\section{Abbreviations}

DEG, Differentially expressed unigenes

RNA-Seq, RNA-sequencing

RPKM, Reads per kilobase per million 